\title{
Micro-CT evaluation of asymmetrical ovine intervertebral disc height loss from surgical approach
}

\section{Ludtka, Christopher}

2017-08

Ludtka , C , Schwan , S , Friedmann , A , Brehm , W , Wiesner , I \& Goehre , F 2017 , ' Micro-CT evaluation of asymmetrical ovine intervertebral disc height loss from surgical approach ' , European Spine Journal , vol. 26 , no. 8 , pp. 2031-2037 . https://doi.org/10.1007/s00586-017-5024-8

http://hdl.handle.net/10138/243404

https://doi.org/10.1007/s00586-017-5024-8

publishedVersion

Downloaded from Helda, University of Helsinki institutional repository.

This is an electronic reprint of the original article.

This reprint may differ from the original in pagination and typographic detail.

Please cite the original version. 


\title{
Micro-CT evaluation of asymmetrical ovine intervertebral disc height loss from surgical approach
}

\author{
Christopher Ludtka $^{1,2} \cdot$ Stefan Schwan ${ }^{1,3}$ - Andrea Friedmann ${ }^{1,3} \cdot$ Walther Brehm $^{3,4}$. \\ Ingo Wiesner ${ }^{5} \cdot$ Felix Goehre ${ }^{5,6}$
}

Received: 29 July 2016/Revised: 16 January 2017/Accepted: 28 February 2017/Published online: 13 March 2017

(C) Springer-Verlag Berlin Heidelberg 2017

\begin{abstract}
Purpose The primary goal of this study is to clearly define and evaluate new intervertebral disc height parameters in analysing the morphological pathology of disc degeneration for application in damage model and regeneration therapy development, as well as applying traditional variables to 3-D characterization methods.

Methods A posterolateral surgical approach was used to induce disc degeneration in an ovine model. At 12-months post-operation, sheep vertebral segments were removed and characterized using micro-CT to evaluate disc height parameters in regard to injury localization.

Results Statistically significant differences between the disc height loss of the left and right side of the disc, consistent with the lateral surgical approach used were seen using the modified average disc height method by Dabbs et al. However, convexity index and the newly proposed
\end{abstract}

Stefan Schwan

stefan.schwan@imws.fraunhofer.de

1 Fraunhofer Institute for Microstructure of Materials and Systems IMWS, Halle (Saale), Germany

2 Department of Chemical and Biomolecular Engineering, University of Tennessee Knoxville, Knoxville, TN, USA

3 Translational Center for Regenerative Medicine, University of Leipzig, Leipzig, Germany

4 Department of Veterinary Medicine, University of Leipzig, Leipzig, Germany

5 Department of General Surgery, BG Bergmannstrost Clinic, Halle, Germany

6 Department of Neurosurgery, University of Helsinki, Helsinki, Finland
Cross Tilt Index did not conclusively demonstrate a difference.

Conclusion Two-dimensional morphological evaluations can be applied in 3-D to provide a more complete picture of disc height loss for injury models. New 3-D parameters that are tailored to the type of surgical approach used should be investigated, with the 9-point system described herein providing a useful basis for derived values. Additionally, the surgical approach chosen when artificially injuring the disc can result in asymmetrical degeneration, as indicated by uneven disc height loss.

Keywords Intervertebral disc height - Micro-computed tomography $\cdot \mu \mathrm{CT} \cdot \mathrm{IVD}$ degeneration - Animal model · Disc herniation

\section{Introduction}

Several quantitative and semi-quantitative measurements are commonly used to assess intervertebral disc degeneration (IDD), such as disc height, histology grade, type II collagen expression, and T2-weighted magnetic resonance imaging (MRI) for nucleus pulposus (NP) hydration [1]. Non-invasive methods such as MRI-derived hydration and CT/MRI disc height are practical for clinical assessments of disc health, but animal and cadaver studies allow for more detailed characterization of the disc joint and tissue. Intervertebral disc (IVD) height in particular is relevant across several areas of interest for IDD, such as developing animal disc degeneration models [2, 3], anatomical modelling for artificial devices [4, 5], and evaluating the efficacy of regenerative therapies $[1,6,7]$.

The most common variables derived from disc height measurements are average disc height, disc height index, and 
disc convexity, which are evaluated at the anteroposterior midline of the disc from MRI, radiographs, or computed tomography (CT). Average disc height is described by Dabbs et al. (herein designated as $\mathrm{DH}_{\mathrm{D}}$ ) as the mean value of the anterior and posterior disc heights and has been adopted for use in other studies, primarily for humans [8-10]. Disc convexity was described by Twomey and Taylor (herein designated as $\mathrm{CI}_{\mathrm{T}}$ ) in a human cadaver study using three measurement points for disc height: anterior, middle, and posterior [11]. They defined the convexity index as the middle disc height divided by the sum of the anterior and posterior disc heights (Table 1). Iterations of convexity index have been used by several studies to evaluate induced degeneration models and the effects of regenerative therapies [10, 12].

Many studies default to utilizing unclearly defined or simplistic measurement methods and do not take advantage of available 3-D characterization methods in preclinical studies. As such, the aim of this study is to define and apply new disc height evaluation methods for use in preclinical studies that clearly define measurements and use 3-D imaging capabilities to, for example, enable evaluation of non-symmetrical degeneration of the IVD based on surgical approach. Many IVD artificial damage models use lateral or frontolateral approaches to induce degeneration $[2,13,14]$, but no evaluations have previously been made on the effect surgical approach can have on the pattern of intervertebral disc height loss in 3-D.

\section{Materials and methods}

\section{Animal model}

This study includes data from 13 Merino sheep, all healthy adult females of average weight and 2 years of age at the time of surgery. Test animals were evaluated for general health and mobility to preclude pre-existing diseases or musculoskeletal deficits.

\section{Surgery protocol}

\section{Intraoperative protocol}

A percutaneous posterolateral approach under C-arm X-ray control was used to access the disc space. A $0.5-\mathrm{cm}$ long

Table 1 Calculation formulas of the common disc parameters

\begin{tabular}{lll}
\hline Method & Formula & Parameters \\
\hline Average disc height [15] & $\mathrm{DH}_{\mathrm{D}}=\frac{A+P}{2}$ & $\begin{array}{l}\text { A-anterior disc height } \\
\mathrm{P} \text {-posterior disc height }\end{array}$ \\
Convexity index [11] & $C_{T}=\frac{C}{A+P}$ & $\begin{array}{l}\text { A-anterior disc height } \\
\text { C-central disc height } \\
\end{array}$ \\
& & $\mathrm{P}$-posterior disc height
\end{tabular}

incision was made at the skin surface as the entry point for reaching the L1/L2 disc, as it was easiest to access using this method. A surgical awl was inserted into the annulus fibrosus to establish the surgical corridor (Fig. 1a). A rongeur was then inserted (Fig. 1b), and a single-punch sequestrum $\left(1-2 \mathrm{~cm}^{3}\right)$ of the NP removed to create mechanical damage (Fig. 1c) and induce degeneration. The incision was closed with two staples, and the surgical site washed with $70 \%$ isopropanol/ethanol and povidoneiodine.

The L2/L3 and L4/L5 segments were evaluated as control levels. L2/L3 was chosen due to its close proximity and anatomy to the damaged disc (L1/L2) and L4/L5 due to being farther from the damage site and any collateral soft tissue damage.

\section{Post-operative protocol}

Each test animal was monitored for 1 week in an indoor recovery enclosure following surgery. After that week, all animals were returned to a standard livestock farm and released for daytime grazing and fully unrestricted movement.

All animals were killed 12 months after surgery. The lumbar spine of each animal was fully excised, cleaned of residual tissue, and frozen at $-20{ }^{\circ} \mathrm{C}$. The spines were then dissected to isolate the $\mathrm{L} 1 / \mathrm{L} 2, \mathrm{~L} 2 / \mathrm{L} 3$, and $\mathrm{L} 4 / \mathrm{L} 5$ motion segments. All segments were refrozen at $-10{ }^{\circ} \mathrm{C}$ and then deep frozen to $-80{ }^{\circ} \mathrm{C}$ for $24 \mathrm{~h}$ before micro-CT analysis.

\section{Micro-CT protocol}

A phoenix nanomelx 180NF (GE, USA) operating with increasing radiation intensity and extended integration times was used to image individual segments. Each 3-D object reconstruction was compiled from 1000 images taken over $360^{\circ}$ rotation using a $400 \mathrm{~ms}$ exposure time, $100 \mathrm{kV}$ voltage, and $135 \mu \mathrm{A}$ current. The resulting voxel size was approximately $25 \mu$.

\section{Image analysis}

The micro-CT scan data from each segment were 3-D rendered using VG StudioMAX 2.2 (Volume Graphics BmgH, Heidelberg, Germany). Each 3-D object was then manually rotated to establish a relative coordinate system such that the axial face of the vertebral body was aligned with the $x y$ plane, with the $y$-axis bisecting the symmetrical halves of each disc in the axial view (Fig. 2a). The view is directed towards the posterior end of the animal; thus, the 'left' side of the disc corresponds to the animal's right side. Threshold values were manually set for each sample so as to most clearly visualize the bone of the endplates. Each disc height 


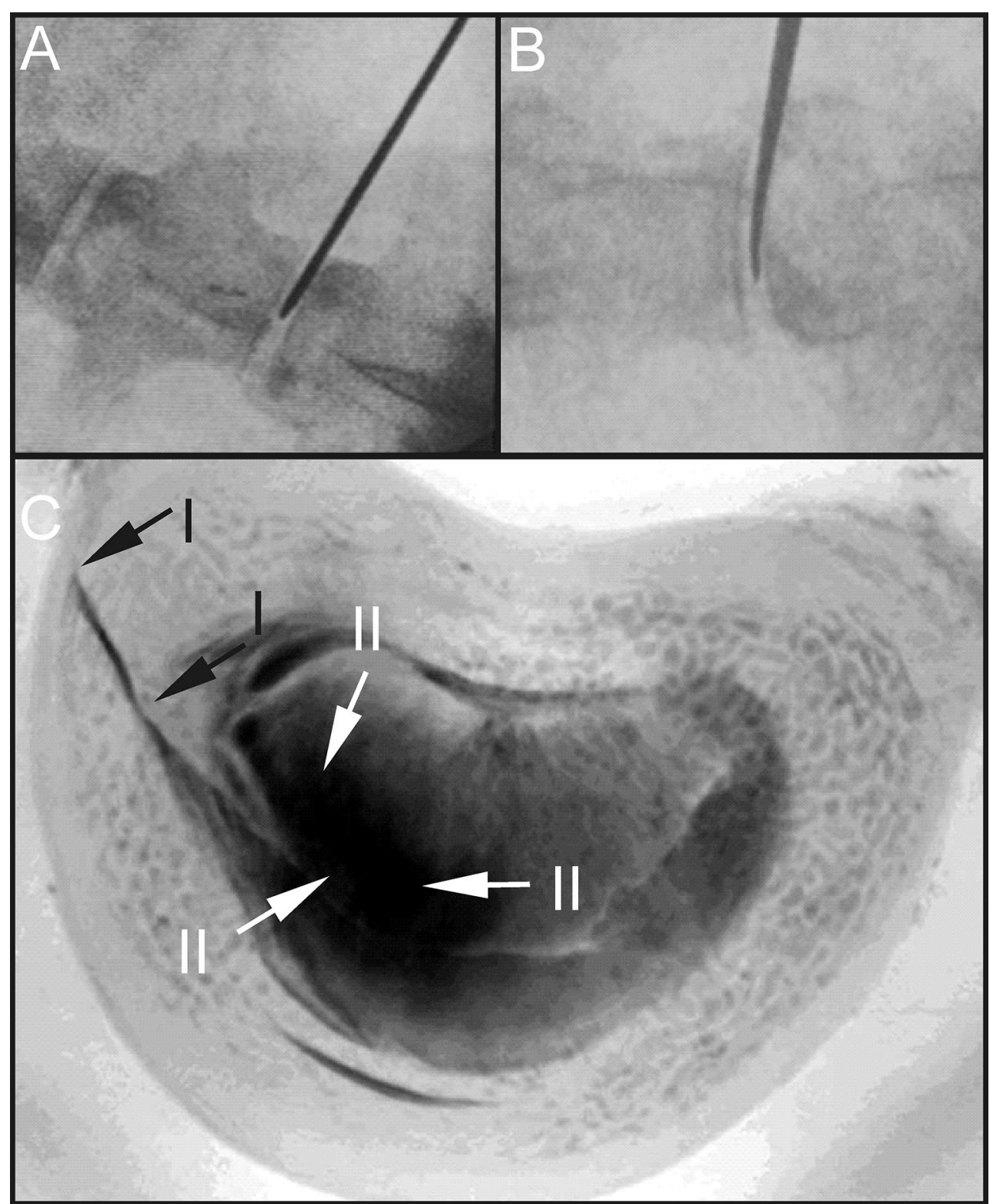

Fig. 1 Intraoperative discography of ovine lumbar spine disc space penetrated by a rongeur (coronal view) and $\mathbf{b}$ a surgical awl (sagittal view) to create mechanical damage to the nucleus pulposus and induce degeneration. c Micro-CT of the disc (in axial view) after

measurement point was labelled according to Fig. $2 b$ based on its location at the intersection of lines defined as anterior/central/posterior and left/middle/right. The nine points are anterior left $(\mathrm{AL})$, anterior middle (AM), anterior right (AR), central left (CL), central middle (CM), central right $(\mathrm{CR})$, posterior left (PL), posterior middle (PM), and posterior right $(\mathrm{PR})$. The in-program distance instruments were used to precisely measure the disc height in a sagittal viewing plane at the anterior minimum, central maximum, and posterior minimum of each disc space (Fig. 3e). The same three measurements (anterior, central, posterior) were then made $5 \mathrm{~mm}$ to the right (Fig. 3f) and left of the midline (Fig. 3d). surgical damage, localized on the 'left side' of the disc (animal's right side). The annulus fibrosus was punctured (black arrows, $I$ ) to access and damage the nucleus pulposus (white arrows, II)

As these six measurements on the right and left sides fall along the same relative $y$-value, they do not necessarily correspond to the local minimums/maximum at that sagittal slice of the disc. This methodology was chosen to ensure comparability between the right and left sides of the disk, as detecting asymmetry requires mirrored coordinates across the plane of symmetry.

\section{Derived values}

We defined and evaluated a new factor: the Cross Tilt Index. We also compared the left and right side of the disc 


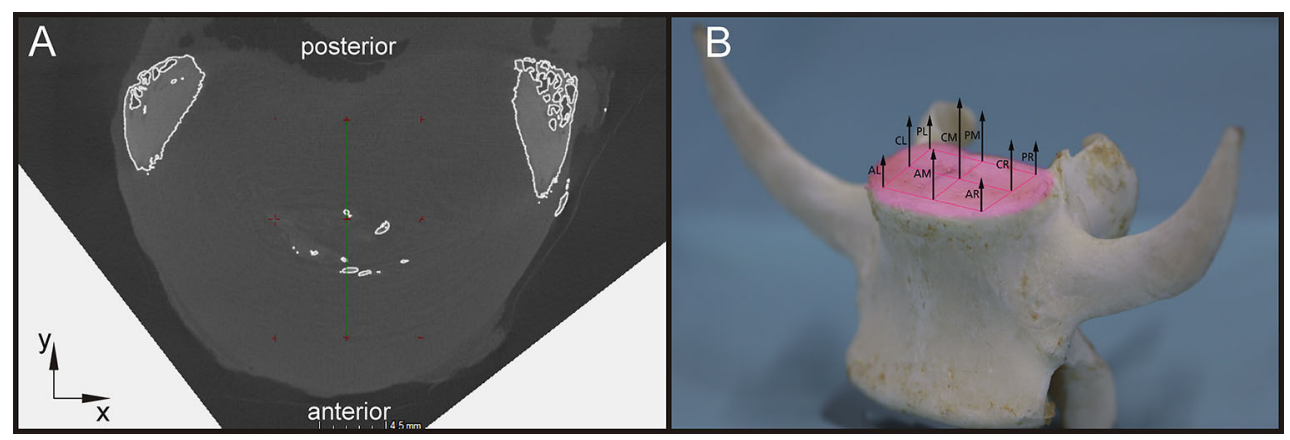

Fig. 2 Proposed disc height measurement points schema. a Micro-CT image of the IVD space in axial view, looking posteriorly. b Each point labelled and illustrated on an excised vertebra

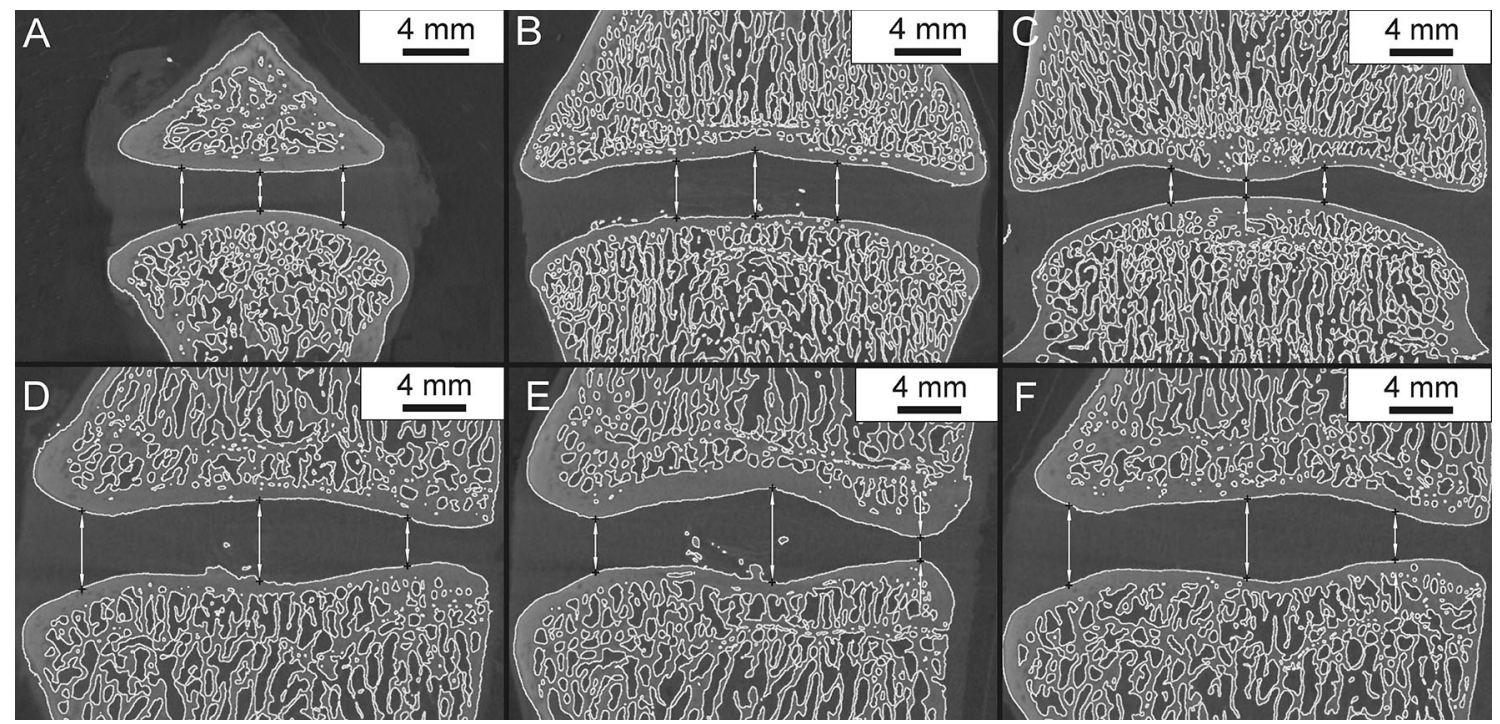

Fig. 3 Micro-computed tomography slices in coronal view of the nine measurement points at the a anterior minimum, b central maximum, and c posterior minimum. Micro-computed tomography slices in the sagittal view of the same nine points at the d left,

using traditional methods including average disc height [8] and convexity index [11]. Values were derived using the nine points collected via micro-CT image analysis. Average disc height was calculated according to Dabbs et al. and convexity index according to Twomey and Taylor. Average disc height is defined by Dabbs et al. as the average of the disc height at the anterior and posterior ends of the IVD space in a sagittal view (Table 1). The convexity index was calculated as the quotient of the central disc height divided by the sum of the anterior and posterior disc heights, all measured in a sagittal view (Table 1). Additionally, a new measurement index is proposed and calculated for assessing diagonal tilt, compared between the AL-PR and AR-PL axes. The intention behind this new measurement, termed the Cross Tilt Index, was to evaluate any diagonal tilt across the IVD, for use in evaluating asymmetrical disc height loss. The Cross Tilt Indices were $\mathbf{e}$ middle, and $\mathbf{f}$ right side of the disc. Note: uneven background in $\mathbf{a}$ is a result of manually realigning the 3 -D generated object to a relative coordinate system

defined as the differences between the disc height measurements at opposite corners (anterior right-posterior left; anterior left-posterior right). The two indices were termed $\mathrm{CTI}_{1}$ and $\mathrm{CTI}_{2}$, respectively (Table 2).

\section{Data analysis}

All statistical analyses were completed using the software OriginPro2015 (OriginLab, Northampton, USA). All data sets demonstrated a normal distribution using the ShapiroWilk normality test with a $95 \%$ confidence interval. Oneway ANOVA tests (Scheffe test with a 95\% confidence interval) were conducted to compare the base measurements between the native and damaged segments for each of the nine disc height measurement locations. The derived variables used to evaluate non-symmetrical degeneration of the disc were also evaluated by one-way ANOVA. 
Table 2 Calculation formula of the newly defined disc parameter

\begin{tabular}{lll}
\hline Method & Formula & Parameters \\
\hline Cross tilt indices & $\mathrm{CTI}_{1}=\mathrm{AR}-\mathrm{PL}$ & $\mathrm{AR}$-anterior right disc height \\
& $\mathrm{CTI}_{2}=\mathrm{AL}-\mathrm{PR}$ & $\mathrm{AL}$-anterior left disc height \\
& & $\mathrm{PR}$-posterior right disc height \\
& $\mathrm{PL}$-posterior left disc height \\
\hline
\end{tabular}

\section{Results}

\section{Base measurements}

Of the nine measurement points for disc height, one-way ANOVA showed that the native and damaged segments differed significantly at the central middle (CM, $p=0.022$ ) and central left (CL, $p=0.030)$ positions (Fig. 4). The average disc height of the CM point (mean $\pm \mathrm{SD}$ ) was $4.02 \pm 0.57 \mathrm{~mm}$ for native segments and $3.60 \pm 0.39 \mathrm{~mm}$ for damaged segments. For the point at $\mathrm{CL}$, the averaged disc heights were $3.52 \pm 0.47 \mathrm{~mm}$ for native segments and $3.20 \pm 0.27 \mathrm{~mm}$ for damaged. The remaining seven points did not show a significant difference between the damaged and native samples based on the $95 \%$ confidence interval used, as seen by the corresponding $p$ values: anterior left (AL, $p=0.198)$, posterior left (PL, $p=0.182$ ), anterior middle (AM, $p=0.141)$, posterior middle $(\mathrm{PM}, p=0.757)$ anterior right $(\mathrm{AR}, p=0.087)$, central right $(\mathrm{CR}, p=0.128)$, and posterior right (PR, $p=0.689$ ).

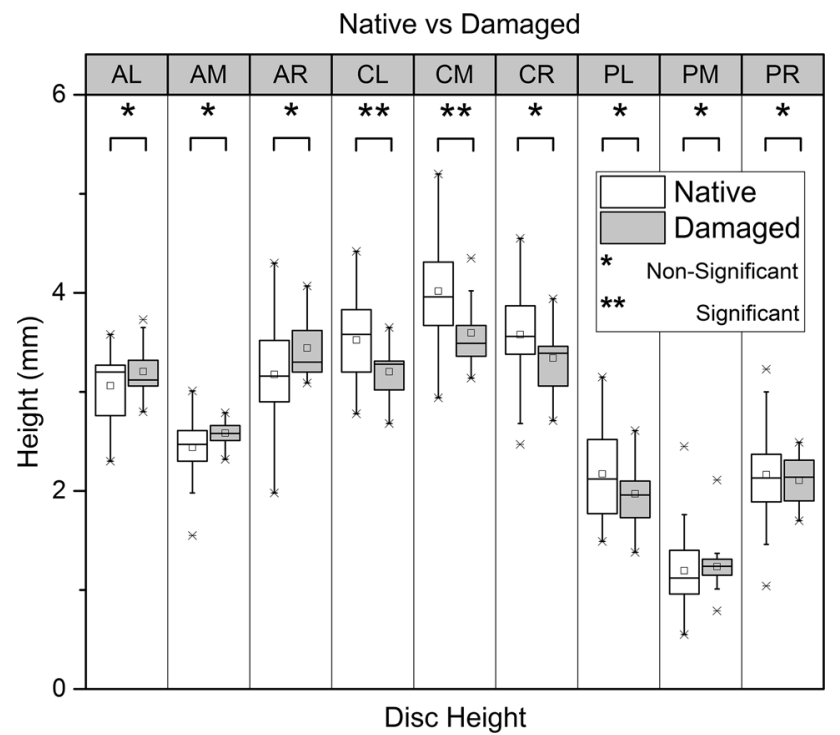

Fig. 4 Boxplot comparing the distribution of disc height measurements between the native (dark shade) and damaged (light shade) segments at each of the nine points of interest. The points are: anterior left (AL), anterior middle (AM), anterior right (AR), central left (CL), central middle $(\mathrm{CM})$, central right $(\mathrm{CR})$, posterior left $(\mathrm{PL})$, posterior middle (PM), and posterior right (PR)

\section{Derived values}

\section{Average disc height}

$\mathrm{DH}_{\mathrm{D}}$ showed a significant difference between the left and right side of the damaged segments $(p=0.028)$, but not of the native segments $(p=0.592)$.

\section{Convexity index}

$\mathrm{CI}_{\mathrm{T}}$ demonstrated no significant difference between the left and right side of damaged segments $(p=0.422)$. There was also no significant difference between left and right convexity for native segments $(p=0.882)$.

\section{Cross tilt index}

The two indices, $\mathrm{CTI}_{1}$ and $\mathrm{CTI}_{2}$, were compared as previously described. The analysis did not show a significant difference between the anterior right to posterior left line $\left(\mathrm{CTI}_{1}\right)$ and the anterior left to posterior right line $\left(\mathrm{CTI}_{2}\right)$ for either the native $(p=0.533)$ or damaged $(p=0.072)$ segments.

\section{Discussion}

This study indicates that the choice of surgical approach can have a significant effect on the pattern of disc height loss and can cause asymmetrical disc height. Disc degeneration has previously been correlated with decreased disc height $[8,10]$; thus, greater disc height loss on one side of the disc may indicate localized degeneration. Micro-CT is a common imaging technique for ex vivo evaluation of the spine [15]. Previous micro-CT studies have also investigated Modic changes in the context of IDD, though typically for naturally occurring degeneration in humans $[16,17]$. These studies investigated micro-architectural parameters of the subchondral bone, as opposed to disc height loss, which compliments this study by providing the context of degenerative changes in the bone compared to the overall IVD joint. The parameters evaluated (bone volume fraction, trabecular thickness, connectivity density) could be used in artificial damage models to assess effects of surgical approach as well. 
Our findings are relevant to standardized IVD damage models in experimental animals as preclinical studies rely on reproducible injury models to evaluate the progression of IVD degeneration, oftentimes through disc height loss. Surgical damage models are ideal compared to chemical and mechanical force models, as the injury is more controlled and reproducible. Several surgical approaches have previously been documented including anterolateral, lateral, posterolateral, and transpedicular approaches $[2,13,14]$, which use either open surgery or percutaneous methods. These various approaches access the disc from different orientations, result in varying amounts of collateral tissue damage, and also utilize different methods (e.g. sharp cutting of the annulus fibrosus, dull puncture of the NP, endplate scraping) to artificially damage the IVD. As such, the degeneration induced within the IVD from this injury may be uneven or localized based on the approach used. Investigating and accounting for the possibility of asymmetrical degeneration is critical in having a comprehensive damage model. Another consideration is evaluating regenerative therapies. Many studies of the efficacy of proposed regenerative IVD treatments use an IVD damage model to compare a standard injury and level of degeneration between the control and experimental groups. To evaluate the regenerative abilities of these treatments, it is critical to fully understand the effects and resultant morphology of the IVD that results from the damage model being used.

Applying $\mathrm{DH}_{\mathrm{D}}$ in 3-D showed this asymmetrical degeneration effect. While the disc height between the left and right sides of the disc differed significantly in average disc height, the convexity indices did not differ between left and right. Therefore, the overall curvature of the IVD and vertebral endplate did not change with respect to the surgical approach employed. This is curious, as previous studies have shown a negative correlation between disc convexity and degeneration $[10,11]$. Interestingly, these same studies have also reported a positive correlation between disc convexity and age. This is at odds with the traditional view of age as a contributing factor to IDD, which has been found to be even more impactful than differences in mechanical load [18, 19]. However, the effect of age is not evaluated in the present study, as all experimental animals were of approximately the same age. Therefore, further investigation of disc convexity in relation to disc degeneration and age, especially in 3-D, would prove valuable.

Additionally, the newly proposed $\mathrm{CTI}_{1}$ and $\mathrm{CTI}_{2}$ did not indicate asymmetrical disc height loss across the two diagonal axes of the disc, though the $p$ value for the damaged sample group was close to the $95 \%$ confidence interval threshold $(p=0.072)$ while the native group had a $p$ value quite far from the significance threshold $(p=0.533)$. One possibility for the lack of conclusive correlations of this method is the surgical approach used in this study. As seen in Fig. 1c, the injury introduced to the disc from a posterolateral approach is localized on the left side of the IVD, entering at an angle through the left side rather than through the posterior left corner. As the Cross Tilt Index was proposed to evaluate differences in disc height between the two diagonal axes of the disc, it may be sensitive to statistically significant differences from an injury localized to a corner of the disc rather than to the side, as would likely be seen in a farposterolateral or far-anterolateral approach.

High-resolution magnetic resonance imaging (MRI) has also been used previously to make 3-D characterizations of the IVD and the relation of certain parameters to degeneration [20]. High-resolution MRI offers a clinical alternative to micro-CT for 3-D visualization of tissues, allowing in vivo characterization and the possibility of clinical diagnosis and evaluation. The primary focus in the relevant literature has been on developing finite element analysis models for the development of personalized and generalized artificial disc implants and creating a full-disc model of degeneration. However, the digitization of the disc in 3-D is typically reduced to several simplified parameters such as disc height, width, anteroposterior depth, and axial area for correlation with Pfirrmann score grading. The derived values detailed in the present study can be applied to high-resolution MRI; however, animal studies do not have the same clinical restrictions that often necessitate MRI in place of micro-CT.

Although IVD height is a common measurement in evaluating IDD and regenerative therapies, the role disc height plays in the degenerative process remains elusive [21, 22]. IDD has a complex aetiology, as evinced by studies indicating numerous possible contributory factors to degeneration including age, gender, mechanical load, trauma, infection, obesity, smoking, occupation, and hormonal and metabolic abnormalities [23]. Additionally, as both damage models and certain naturally occurring spine injuries are directional in nature (e.g. herniation), it is important to understand how the localization of damage affects the gross morphology of the disc space and degeneration process. Three-dimensional assessment of disc degeneration offers a more complete picture of degenerative changes in the disc and should be taken advantage of in animal and cadaver studies. Additionally, other characterization techniques can be evaluated on a localized basis to ascertain if other degenerative symptoms such as loss of NP hydration or type II collagen expression are localized factors.

\section{Conclusions}

The surgical approach taken when accessing the IVD space and creating an artificial injury can cause asymmetrical disc degeneration as seen by localized disc height loss. 
Two-dimensional derived values can be applied in 3-D for imaging techniques such as micro-CT to evaluate the presence of such effects. In this study, average disc height was seen to correlate with the site of the injury, with greater disc height loss on the disc's damaged side. Further 3-D investigations of localized disc damage should be conducted, particularly in the context of representative injury models and regenerative therapy evaluations. These may need to be adjusted to best assess any disc height loss based on the surgical approach used.

Acknowledgements The authors would like to thank M. Menzel and W. Petzold for technical assistance. The work presented in this paper was made possible by funding from the German Federal Ministry of Education and Research (BMBF, 1315883) and Whitaker International Program.

\section{Compliance with ethical standards}

Conflict of Interest All the authors declare that they have no conflict of interest.

\section{References}

1. Wang Z, Perez-Terzic CM, Smith J et al (2015) Efficacy of intervertebral disc regeneration with stem cells-a systematic review and meta-analysis of animal controlled trials. Gene 564:1-8. doi:10.1016/j.gene.2015.03.022

2. Oehme D, Goldschlager T, Rosenfeld J et al (2012) Lateral surgical approach to lumbar intervertebral discs in an ovine model. Sci World J 2012:873726. doi:10.1100/2012/873726

3. Wilke HJ, Kettler A, Wenger KH, Claes LE (1997) Anatomy of the sheep spine and its comparison to the human spine. Anat Rec 247:542-555. doi:10.1002/(SICI)1097-0185(199704)247:4<542

4. van der Houwen EB, Baron P, Veldhuizen AG et al (2010) Geometry of the intervertebral volume and vertebral endplates of the human spine. Ann Biomed Eng 38:33-40. doi:10.1007/ s10439-009-9827-6

5. Korez R, Likar B, Pernus F, Vrtovec T (2014) Parametric modeling of the intervertebral disc space in 3D: application to CT images of the lumbar spine. Comput Med Imaging Graph 38:596-605. doi:10.1016/j.compmedimag.2014.04.008

6. Ghosh P, Moore R, Vernon-Roberts B et al (2012) Immunoselected STRO-3(+) mesenchymal precursor cells and restoration of the extracellular matrix of degenerate intervertebral discs laboratory investigation. J Neurosurg Spine 16:479-488. doi:10. 3171/2012.1.SPINE11852

7. Hiyama A, Mochida J, Iwashina T et al (2008) Transplantation of mesenchymal stem cells in a canine disc degeneration model. J Orthop Res 26:589-600. doi:10.1002/jor.20584

8. Dabbs VM, Dabbs LG (1990) Correlation between disk height narrowing and low-back pain. Spine 15:1366-1369. doi:10.1097/ 00007632-199012000-00026
9. Frobin W, Brinckmann P, Biggemann M et al (1997) Precision measurement of disc height, vertebral height and sagittal plane displacement from lateral radiographic views of the lumbar spine. Clin Biomech 12:S1-S63. doi:10.1016/S0268-0033(96)00067-8

10. Pfirrmann CWA, Metzdorf A, Elfering A et al (2006) Effect of aging and degeneration on disc volume and shape: a quantitative study in asymptomatic volunteers. J Orthop Res 24:1086-1094. doi:10.1002/jor.20113

11. Twomey LT, Taylor JR (1985) Age-changes in the lumbar articular triad. J Anat 142:226-227

12. Holguin N, Muir J, Rubin C, Judex S (2009) Short applications of very low-magnitude vibrations attenuate expansion of the intervertebral disc during extended bed rest. Spine J 9:470-477. doi:10.1016/j.spinee.2009.02.009

13. Guder E, Hill S, Kandziora F, Schnake KJ (2009) Partial nucleotomy of the ovine disc as an in vivo mode for disc degeneration. Z Orthop Unfall 147:52-58. doi:10.1055/s-20081039139

14. Melrose J, Roberts S, Smith S et al (2002) Increased nerve and blood vessel ingrowth associated with proteoglycan depletion in an ovine anular lesion model of experimental disc degeneration. Spine 27:1278-1285. doi:10.1097/00007632-200206150-00007

15. Maerz T, Newton MD, Kristof K et al (2014) Three-dimensional characterization of in vivo intervertebral disc degeneration using EPIC- $\mu$ CT. Osteoarthritis and Cartilage 22:1918-1925. doi:10. 1016/j.joca.2014.08.006

16. Perilli E, Parkinson IH, Truong L-H et al (2015) Modic (endplate) changes in the lumbar spine: bone micro-architecture and remodelling. Eur Spine J 24:1926-1934. doi:10.1007/s00586014-3455-Z

17. Rutges JP, Jagt van der OP, Oner FC et al (2011) Micro-CT quantification of subchondral endplate changes in intervertebral disc degeneration. Osteoarthr Cartil 19:89-95. doi:10.1016/j.joca. 2010.09.010

18. Battie MC, Videman T, Parent E (2004) Lumbar disc degeneration-epidemiology and genetic influences. Spine 29:2679-2690. doi:10.1097/01.brs.0000146457.83240.eb

19. Cheung KMC, Karppinen J, Chan D et al (2009) Prevalence and pattern of lumbar magnetic resonance imaging changes in a population study of one thousand forty-three individuals. Spine 34:934-940. doi:10.1097/BRS.0b013e3181a01b3f

20. Peloquin JM, Yoder JH, Jacobs NT et al (2014) Human L3L4 intervertebral disc mean 3D shape, modes of variation, and their relationship to degeneration. J Biomech 47:2452-2459. doi:10. 1016/j.jbiomech.2014.04.014

21. Adams MA, Roughley PJ (2006) What is intervertebral disc degeneration, and what causes it? Spine 31:2151-2161. doi:10. 1097/01.brs.0000231761.73859.2c

22. Buckwalter JA (1995) Spine update-aging and degeneration of the human intervertebral disc. Spine 20:1307-1314

23. Chan WCW, Sze KL, Samartzis D et al (2011) Structure and biology of the intervertebral disk in health and disease. Orthop Clin N Am 42:447. doi:10.1016/j.ocl.2011.07.012 